\title{
Conjugated Versus "Free" Acidic Metabolites of Catecholamines in Random Urine Samples: Significance for the Diagnosis of Neuroblastoma
}

\author{
MENDEL TUCHMAN AND JOEL S. STOECKELER
}

Divisions of Metabolism and Health Computer Science, Departments of Pediatrics and Laboratory Medicine and Pathology, University of Minnesota, Minneapolis, Minnesota 55455

\begin{abstract}
Random urine samples were obtained from 31 patients with neuroblastoma (newborn to $8 \mathrm{yr}$ of age) and from 100 children without this tumor (newborn to 10 yr). The urine samples were studied for the presence of sulfate and glucuronide conjugates of homovanillic (HVA), dihydroxyphenylacetic, vanilmandelic, and vanillactic acids. The urinary concentrations of these acids were determined by capillary gas-chromatography before and after enzymatic treatment with glucuronidase and sulfatase. Concentrations of the "free" fraction and "total" urinary content of these acids were determined using the results from untreated and treated urines respectively. Age-related reference values were established for children without neuroblastoma. Fractions of the total content of urinary HVA (18-39\%) and dihydroxyphenylacetic acid (36-66\%) were excreted as glucuronides and/or sulfates by the control group, with the highest conjugated fractions found in the urine of young infants (0-3 months). Vanilmandelic was excreted mainly as "free" acid (unconjugated), whereas vanillactic acid was undetectable in almost all control samples. Patients with neuroblastoma also excreted a fraction of these acids as glucuronide and/or sulfate conjugates, (25\% of urinary HVA, 39\% of dihydroxyphenylacetic acid and $45 \%$ of vanillactic acid) whereas vanilmandelic acid was excreted only as "free" in controls. Determination of "total" rather than "free" urinary HVA was diagnostic in one neuroblastoma patient with borderline "free" HVA levels, whereas determination of "free" or "total" dihydroxyphenylacetic acid and vanillactic acid did not improve the diagnostic sensitivity in the cases examined. We conclude that it may be clinically useful to determine "total" urinary HVA in patients with borderline "free" HVA levels who are suspected of having neuroblastoma. (Pediatr Res 23: 576-579, 1988)
\end{abstract}

\section{Abbreviations}

HVA, homovanillic acid

DOPAC, 3,4-dihydroxyphenylacetic acid

VMA, vanilmandelic acid

VLA, vanillactic acid

UCr, urinary creatinine concentration
Most children with neuroblastoma excrete higher than normal amounts of catecholamine metabolites in their urine (1-3). The measurement of urinary catecholamine metabolites has been used routinely to establish or confirm the diagnosis of this tumor $(4,5)$. Three main groups of catecholamine metabolites have been found in elevated concentrations in the urines of neuroblastoma patients 1 ) acidic derivatives including HVA, VMA, DOPAC, and VLA; 2) Alcoholic derivatives including: 4-hydroxy-3-methoxyphenylethylglycol(HMPG) 3,4 dihydroxyphenylethylglycol; and 3) metanephrines. Of the metabolites listed above, HVA and VMA are used most often as neuroblastoma markers because they are excreted most consistently in elevated amounts by patients with neuroblastoma (5). Of patients with neuroblastoma $92 \%$ have elevated concentrations of HVA and/ or VMA in random urine samples at diagnosis (6). The use of prolonged urine collections rather than random urine samples is not advantageous in respect of diagnostic sensitivity $(7,8)$.

Some catecholamine metabolites such as HVA, DOPAC and 4-hydroxy-3-methoxyphenylethylglycol appear in the urine as free acids, and as sulfate and glucuronide conjugates (9-12). Routine methodologies measure only the "free" portion of these metabolites in the urine whereas the conjugated portions remain unaccounted for unless these conjugates are chemically or enzymatically cleaved before analysis (13). This study investigated the clinical implications of measuring the "free" fraction versus the total content of HVA, VMA, DOPAC, and VLA in random urine samples for the use in the diagnosis of neuroblastoma. Agematched normal values were established for "total" and "free" portions of these acids and compared to values obtained from patients with neuroblastoma at diagnosis.

\section{PATIENT POPULATION}

Random urine samples obtained at diagnosis from patients with neuroblastoma aged newborn to $8 \mathrm{yr}$ were studied. The diagnosis of neuroblastoma was confirmed histologically in all patients. These patients were at various clinical disease stages at the time of diagnosis. Random urine samples were obtained and studied in a control pediatric population (age newborn to $10 \mathrm{yr}$ ) which included healthy children and pediatric patients in whom the diagnosis of neuroblastoma had been excluded. The control population consisted of five different age groups with 20 individuals included in each group. The urine samples were delivered to the laboratory immediately after collection or were frozen at $-20^{\circ} \mathrm{C}$ until delivery or analysis.

\section{MATERIALS AND METHODS}

Correspondence and reprint requests Mendel Tuchman, M.D., Department of Pediatrics, University of Minnesota, Box 400 UMHC, Harvard Street at East River Road, Minneapolis, MN 55455.

Supported by a grant from the Minnesota Medical Foundation and by Grant LM07041 from the National Institute of Health.
Chemicals. HVA, VMA, DOPAC, VLA, 3,4-dihydroxybenzoic acid, resorcinol, and Helix Pomatia-derived sulfatase + glucuronidase type H-2 were purchased from Sigma Chemical 
Company, St. Louis, MO. Ethylacetate and diethylether were purchased from Spectrum Chemical Manufacturing Corp, Redondo Beach, CA. N,O bis-(trimethylsilyl) trifluoroacetamide + $10 \%$ trimethylchlorosilane (Regisil RC-3) was purchased from Regis Chemical Company, Morton Grove, IL.

Analysis of "total" and "free" HVA, VMA, DOPAC, and VLA. $\mathrm{UCr}$ was determined on each urine sample by the method of Folin and $\mathrm{Wu}(14)$. Two urine aliquots of $4 \mathrm{ml}$ of each sample were placed into separate glass tubes (if less urine was available, the total volume was adjusted to $4 \mathrm{ml}$ with deionized water). One $\mathrm{ml}$ of $2.5 \mathrm{M}$ sodium-acetate buffer $\mathrm{pH} 5.3$ containing $0.2 \%$ resorcinol (an antiseptic, to prevent bacterial growth during incubation) was added to each urine aliquot; and the $\mathrm{pH}$ was readjusted to 5.3 with $1 \mathrm{~N} \mathrm{NaOH}$ or $1 \mathrm{M}$ acetic acid solution. Ten thousand units of glucuronidase +0.5 Units sulfatase were added to only one of the two tubes. Both tubes were capped and placed in a $37^{\circ} \mathrm{C}$ water bath for $18 \mathrm{hr}$. At the end of the incubation, $100 \mu \mathrm{g}$ of 3,4-dihydroxybenzoic acid (internal standard) were added to each tube. The $\mathrm{pH}$ was adjusted to less than 1 with $5 \mathrm{~N} \mathrm{HCl}$ solution, and $2 \mathrm{~g}$ of $\mathrm{NaCl}$ were added. The organic acids were extracted from both tubes and prepared for capillary gas chromatographic analysis as described earlier (8). Gas chromatographic conditions for separation and identification of the acids, calculation of the amounts according to internal standard calibration, and normalization of the results to the $\mathrm{UCr}$ were identical to the method described for the determination of urinary HVA and VMA (15). In certain samples containing very small concentrations of DOPAC, it was necessary to separate the acids on a non polar 5\% methylsilicone capillary column (Ultra I Hewlett-Packard Co., Avondale, PA) to avoid interference with DOPAC separation by hippuric acid. In those instances quantitation was done according to internal standard calibration performed on the Ultra I column. The results of the acidic metabolite levels obtained from the urine aliquot pretreated with glucuronidase/sulfatase enzyme represented the "total" amounts of the respective acid, whereas those obtained from the nontreated samples represented the "free" portion of the acid.

Analysis of results. Normal values (mean and 95\% confidence intervals of the mean) of "total" and "free" levels of HVA, VMA, DOPAC, and VLA in random urine samples were established in five age groups. "Total" urinary levels of each acid were compared to the levels of "free" acid in the patients with neuroblastoma and in the control group using a one tail student's paired $t$ test (16). The statistical analysis identified the significant differences between the "free" and "total" values in order to assess which of the urinary acids is conjugated to sulfate and/or glucuronate. The degree of conjugation, its variability and age dependence in the control population were analyzed. Results obtained from the control group were compared to those observed in samples of patients with neuroblastoma in order to assess differences in the magnitude of conjugation between controls and patients with neuroblastoma. The clinical implications of measuring "total" versus "free" concentrations of the acidic metabolites of catecholamines in random urine samples for the diagnosis of neuroblastoma were evaluated.

\section{RESULTS}

The extraction efficiencies of "free" HVA, DOPAC, VMA, and VLA from urine by the method used in this study were 95 , 95,75 , and $85 \%$, respectively, and their limits of detection about $0.1 \mu \mathrm{g} / \mathrm{mg}$ UCr. This study was performed with the assumption that conjugated acids are not extractable by the solvent extraction method, and therefore the sulfate and glucuronide conjugates of these acids can be accounted for by our methodology only after cleavage of the conjugate.

Table 1 summarizes the "free" and "total" levels of HVA, DOPAC, VMA, and VLA in random urine samples obtained from children without neuroblastoma. Consistently higher amounts of HVA and DOPAC have been recovered from the urine samples treated with glucuronidase + sulfatase compared to the untreated samples. The results obtained for VMA and VLA did not show a similar pattern. Table 2 shows statistically significant differences between the "free" fraction and "total" content of HVA and DOPAC in the various age groups, whereas no significant differences have been observed for VMA and VLA. A possible exception is the 0 - to 3-month age group where a significant difference between "free" and "total" urinary VMA has been observed.

Similar results were obtained in patients with neuroblastoma for HVA and DOPAC (Table 3). In these children also, there was no statistically significant difference between the "free" and "total" amount of urinary VMA. However, for VLA there was a significant difference between the "free" and "total" values. These results indicate that HVA and DOPAC are excreted both as "free" and conjugated acids in urine of normal children and children with neuroblastoma. VMA was not excreted in a conjugated form by either group except in the first months of life, whereas an appreciable fraction of VLA was excreted in a conjugated form by some patients with neuroblastoma but not by healthy children. The fraction of the total urinary content of HVA, DOPAC and VLA excreted as sulfate and glucuronide conjugates is shown in Table 4.

Young babies appear to excrete a higher fraction of these acids in a conjugated form when compared to older children. The mean conjugated fraction of HVA in urine of patients with neuroblastoma $(25 \%)$ was similar to the controls $(18-39 \%)$ whereas the conjugated fraction of DOPAC was somewhat higher

Table 2. Level of significance of differences (p) by one-tail paired student's t test between means of free fraction and total content of HVA, DOPAC, VMA, and VLA in random urine samples of children without neuroblastoma

\begin{tabular}{lcccc}
\hline Age & HVA & DOPAC & VMA & VLA \\
\hline 0-3 mo & 0.001 & 0.018 & 0.017 & 0.055 \\
3-12 mo & 0.000 & 0.007 & 0.499 & 0.388 \\
$1-2 \mathrm{yr}$ & 0.001 & 0.042 & 0.090 & 0.085 \\
$2-5 \mathrm{yr}$ & 0.000 & 0.005 & 0.458 & 0.263 \\
$5-10 \mathrm{yr}$ & 0.001 & 0.003 & 0.088 & 0.083 \\
\hline
\end{tabular}

Table 1. "Free" and "Total" HVA, DOPAC, VMA, and VLA in random urine samples of children without neuroblastoma*, $\dagger$

\begin{tabular}{lccccccccc}
\hline \multicolumn{1}{c}{ Age } & HVA $(\mathrm{f}) \ddagger$ & HVA $(\mathrm{t}) \S$ & DOPAC $(\mathrm{f})$ & DOPAC $(\mathrm{t})$ & VMA $(\mathrm{f})$ & VMA $(\mathrm{t})$ & VLA $(\mathrm{f})$ & VLA $(\mathrm{t})$ & $n$ \\
\hline 0-3 mo & $15.5 \pm 3.2$ & $26.8 \pm 6.8$ & $0.8 \pm 1.0$ & $6.5 \pm 5.5$ & $9.0 \pm 3.9$ & $11.9 \pm 4.5$ & $1.3 \pm 1.8$ & $2.3 \pm 3.1$ & $15-20$ \\
$3-12 \mathrm{mo}$ & $15.9 \pm 2.3$ & $21.8 \pm 4.0$ & $0.6 \pm 0.6$ & $2.9 \pm 2.0$ & $13.6 \pm 2.7$ & $13.6 \pm 2.9$ & $0.2 \pm 0.4$ & $0.3 \pm 0.6$ & $18-20$ \\
$1-2 \mathrm{yr}$ & $19.4 \pm 10.0$ & $25.3 \pm 10.3$ & $1.5 \pm 1.2$ & $4.3 \pm 2.7$ & $13.9 \pm 7.1$ & $16.2 \pm 8.4$ & $0.0 \pm 0.0$ & $0.1 \pm 0.0$ & $15-20$ \\
$2-5 \mathrm{yr}$ & $9.4 \pm 1.4$ & $11.4 \pm 1.6$ & $1.9 \pm 0.8$ & $3.2 \pm 1.0$ & $8.8 \pm 4.9$ & $8.7 \pm 4.5$ & $0.5 \pm 0.6$ & $0.5 \pm 0.8$ & $13-20$ \\
$5-10 \mathrm{yr}$ & $5.8 \pm 1.2$ & $7.5 \pm 1.6$ & $1.8 \pm 1.0$ & $3.1 \pm 1.2$ & $4.8 \pm 1.0$ & $5.6 \pm 1.2$ & $0.1 \pm 0.2$ & $0.2 \pm 0.2$ & $18-20$ \\
\hline
\end{tabular}

* Levels below the limit of detection $(<0.1 \mu \mathrm{g} / \mathrm{mg} \mathrm{UCr})$ were calculated as zero values.

$\dagger$ Mean and $95 \%$ confidence intervals for the mean in $\mu \mathrm{g} / \mathrm{mg} \mathrm{UCr}$.

$\ddagger$ Free fraction.

$\S$ Total content. 
Table 3. "Free" and "Total" HVA, DOPAC, VMA, and VLA in random urine samples of children with neuroblastoma* $\dagger$

\begin{tabular}{|c|c|c|c|c|c|c|c|c|c|}
\hline Age & HVA (f) $\ddagger$ & HVA $(\mathrm{t}) \S$ & DOPAC (f) & DOPAC $(t)$ & VMA (f) & VMA (t) & VLA (f) & VLA (t) & $n$ \\
\hline $0-3 \mathrm{mo}$ & 103.9 & 149.9 & 4.6 & 7.6 & 162.8 & 156.8 & 0.2 & 2.1 & 7 \\
\hline $3-12 \mathrm{mo}$ & 127.5 & 184.7 & 6.1 & 23.0 & 75.3 & 91.6 & 0 & 0 & 8 \\
\hline $1-2 \mathrm{yr}$ & 61.3 & 91.6 & 10.4 & 20.9 & 50.6 & 56.5 & 0.2 & 0.3 & 4 \\
\hline $2-5 \mathrm{yr}$ & 130.1 & 157.7 & 16.4 & 19.0 & 101.6 & 99.5 & 1.6 & 3.7 & 10 \\
\hline $5-10 y r$ & 200.1 & 225.0 & 30.1 & 34.1 & 162.9 & 158.4 & 4.4 & 6.3 & 2 \\
\hline All & 119.2 & 158.7 & 11.5 & 19.2 & 106.0 & 108.7 & 1.2 & 2.1 & 31 \\
\hline$p \|$ & \multicolumn{2}{|c|}{0.001} & \multicolumn{2}{|c|}{0.019} & \multicolumn{2}{|c|}{0.286} & \multicolumn{2}{|c|}{0.013} & \\
\hline
\end{tabular}

* Levels below the limit of detection $(<0.1 \mu \mathrm{g} / \mathrm{mg} \mathrm{UCr})$ were not included in the calculation of the mean values.

$\dagger$ Mean in $\mu \mathrm{g} / \mathrm{mg} \mathrm{UCr}$.

$\ddagger$ Free fraction.

$\S$ Total content.

|| Significance levels of the differences between the mean "free" and "total" values (all patients excluding those with undetectable values).

Table 4. Conjugated fractions of HVA, DOPAC, and VLA in random urine samples of children with neuroblastoma and controls*

\begin{tabular}{|c|c|c|c|c|c|c|}
\hline & HVA & $(n)$ & DOPAC & $(n) \dagger$ & VLA & $(n)$ \\
\hline Neuroblastoma & $24.5 \pm 5.0$ & (31) & $39.2 \pm 10.6$ & $(21)$ & $45.3 \pm 19.6$ & (5) \\
\hline \multicolumn{7}{|l|}{ Controls } \\
\hline 0-3 mo & $38.7 \pm 6.5$ & (16) & $66.3 \pm 18.6$ & (4) & $31.5 \pm 12.7$ & (3) \\
\hline $3-12 \mathrm{mo}$ & $27.1 \pm 7.6$ & (15) & $56.0 \pm 29.6$ & (5) & ND $\ddagger$ & \\
\hline $1-2 \mathrm{yr}$ & $25.7 \pm 7.3$ & (19) & $45.1 \pm 28.0$ & (6) & ND & \\
\hline $2-5 \mathrm{yr}$ & $17.7 \pm 2.9$ & (20) & $36.2 \pm 11.6$ & (10) & ND & \\
\hline $5-10 \mathrm{yr}$ & $23.1 \pm 9.2$ & (17) & $41.6 \pm 7.1$ & $(8)$ & ND & \\
\hline
\end{tabular}

* Percent, mean, and $95 \%$ confidence intervals for the mean.

+ Undetectable levels of free fraction or total content were not included in the calculations.

$\ddagger$ Not detectable in any sample.

in controls (36-66\%) compared to the patients (39\%). One patient with neuroblastoma had normal "free" levels of HVA; however, "total" HVA levels were also normal. Two patients had borderline ( 2 SD above the mean) levels of "free" HVA. In one of these patients the "total" HVA level was clearly above normal. Thirteen patients with neuroblastoma had normal levels of "free" DOPAC. In three of these patients "total" DOPAC levels were elevated. The "total" DOPAC level showed excellent correlation with the HVA levels and in none of the three patients with normal or borderline "free" HVA and VMA levels was the "total" DOPAC level elevated.

Urinary "free" VLA was detected only in eight patients with neuroblastoma whereas "total" VLA was detected in 12 patients. All but two "total" VLA values were above normal. Moreover, VLA was not elevated in the three patients with normal or borderline HVA and VMA levels.

\section{DISCUSSION}

The histological diagnosis of neuroblastoma may be difficult without the demonstration of excessive concentrations of catecholamines and their metabolites in blood or urine of the patients. Measurement of acidic metabolites of catecholamines in the urine is used routinely for the diagnosis of neuroblastoma. However, the extraction methodologies used do not usually recover the hydrophilic sulfate and glucuronide conjugates of these acids. Extraction of the conjugates would have resulted in overestimation of the "free" fraction because derivatization for gas chromatography would lead to deconjugation (17). The presence of DOPAC conjugates in human urine was reported by O'Gorman et al. (9) in patients receiving L-DOPA. Other investigators showed that a significant portion of HVA is excreted also in a conjugated form $(18-20)$. VLA is found in elevated concentrations in the urine of some patients with neuroblastoma $(21,22)$; however, the presence of VLA conjugates has not been studied. Our study reports pediatric age-matched reference values for "free" and "total" DOPAC in random urine sample, which are currently unavailable. Our results confirm the observations of other investigators that a significant portion of urinary HVA and DOPAC is conjugated to glucuronate and sulfate. The data obtained herein suggest that VLA is excreted largely in a bound form especially in patients with neuroblastoma in whom increased VLA production occurs. The clinical implications of the above finding are addressed herein. DOPAC, which is a metabolite of DOPA and dopamine, appeared in elevated concentrations in the urine of most patients with neuroblastoma. However, all these patients also had elevated concentrations of HVA. Thus, determination of urinary DOPAC will probably not add to the diagnostic sensitivity of HVA and VMA analysis. If DOPAC is to be used clinically, "total" rather than "free" DOPAC should be determined because a major portion of urinary DOPAC is conjugated. VLA, a metabolite of DOPA, is elevated in the urine of patients with neuroblastoma and has been measured to diagnose this tumor (22). As DOPAC, it correlated with HVA levels, and its determination does not seem to offer an advantage over the determination of HVA. In cases where "free" urinary HVA level is borderline, determination of total content of urinary HVA may be of diagnostic value. In such cases it may be useful also to measure VLA, however, we could not demonstrate this in our study.

\section{REFERENCES}

1. Bell M, Steward JK 1961 Neuroblastoma and catecholamine excretion. Lancet $1: 1288$

2. Bohuon C 1968 Catecholamine metabolism in neuroblastoma. J Pediatr Surg 3:1 14-118

3. LaBrosse EH, Com-Nougué C, Zucker JM, Comoy E, Bohuon C, Lemerle J, Schweisguth O 1980 Urinary excretion of 3-methoxy-4-hydroxymandelic acid and 3-methoxy-4-hydroxyphenylacetic acid by 288 patients with neuroblastoma and related neural crest tumors. Cancer Res 40:1995-2001

4. Schweisguth O 1968 Excretion of catecholamine metabolites in urine of neuroblastoma patients. J Pediatr Surg 3:118-120

5. Hinterberger H, Bartholomew RJ 1969 Catecholamines and their acidic metabolites in urine and in tumor tissue in neuroblastoma ganglioneuroma and pheochromocytoma. Clin Chim Acta 23:169-175

6. Tuchman M, Ramnaraine ML, Woods WG, Krivit W 1987 Three years of 
experience with random urinary homovanillic and vanillylmandelic acid levels in the diagnosis of neuroblastoma. Pediatrics 79:203-205

7. Tuchman M, Morris CL, Ramnaraine ML, Bowers LD, Krivit W 1985 Value of random urinary homovanillic acid and vanillylmandelic acid levels in the diagnosis and management of patients with neuroblastoma: comparison with 24-hour urine collections. Pediatrics 75:324-328

8. Tuchman M, Robison LL, Maynard RC, Ramnaraine ML, Krivit W 1985 Assessment of the diurnal variations in urinary homovanillic acid and vanillylmandelic acid excretion for the diagnosis and follow up of patients with neuroblastoma. Clin Biochem 18:176-179

9. O'Gorman LP, Borud O, Khan IA, Gjessing LR 1970 The metabolism of L3,4-dihydroxyphenylalanine in man. Clin Chim Acta 29:111-119

10. Smith ERB, Weil-Malherbe H 1971 On the occurrence of glusulase-hydrolyzable conjugates of 3,4-dihydroxyphenylacetic and homovanillic acids in human urine. Clin Chim Acta 35:505-507

11. Karoum F, Lefêvre H, Bigelow LB, Costa E 1973 Urinary excretion of 4hydroxy-3-methoxyphenylglycol and 4-hydroxy-3-methoxyphenylethanol in man and rat. Clin Chim Acta 43:127-137

12. Muskiet FAJ, Groen J 1979 Urinary excretion of conjugated homovanillic acid, 3,4-dihydroxyphenylacetic acid, p-hydroxyphenylacetic acid, and vanillic acid by persons on their usual diet and patients with neuroblastoma. Clin Chem 25:1281-1284

13. Ito S, Kato T, Maruta K, Jimbow K, Fujita K 1985 "Total" acidic metabolites of catecholamines in urine as determined by hydrolysis with hydriodic acid and liquid chromatography: Application to patients with neuroblastoma and melanoma. Clin Chem 31:1185-1188

14. Folin O, Wu H 1947 in Hawk PB, Oser BL, Summerson WH (eds) Practical Physiological Chemistry. Blakiston, Philadelphia, pp 839-842

15. Tuchman M, Auray-Blais C, Ramnaraine MLR, Neglia J, Krivit W, Lemieux B 1987 Determination of homovanillic and vanillylmandelic acids from dried filter paper samples: assessment of potential methods for neuroblastoma screening. Clin Biochem 20:173-177

16. Hogg RV, Tanis EA 1983 Probability and Statistical Inference. Maximillian Publishing Co. Inc., New York, pp 345-349

17. Murray S, Baillie TA 1979 Direct derivatization of sulphate esters for analysis by gas chromatography-mass spectrometry. Biomed Mass Spectrom 6:8289

18. Goodall McC, Alton H 1968 Metabolism of 3-hydroxytyramine (dopamine) in human subjects. Biochem Pharmacol 17:905-914

19. Goodall McC, Alton H 1969 Dopamine (3-hydroxytyramine) replacement and metabolism in sympathetic nerve and adrenal medullary depletions after prolonged thermal injury. J Clin Invest 48:1761-1767

20. Goodall McC, Alton H 1969 Dopamine (3-hydroxytyramine) metabolism in parkinsonism. J Clin Invest 48:2300-2308

21. Gjessing LR 1968 Biochemistry of functional neural crest tumors. Advan Clin Chem 11:81-131

22. Wadman SK, Ketting D, Voûte PA 1976 Gas chromatographic determination of urinary vanilglycolic acid, vanilglycol, vanilacetic acid and vanillactic acid-chemical parameters for the diagnosis of neurogenic tumours and evaluation of their treatment. Clin Chim Acta 72:49-68 